\title{
Maintaining Consistency between Caches to Improve Overall Performance in Wireless Sensor Networks
}

\author{
Harish Kumar \\ Department of Electronics \& \\ Communication Engineering \\ Lovely Professional University Phagwara, Punjab \\ (India)
}

\begin{abstract}
Wireless Sensor Network (WSN) is an application based network. Wireless Sensor Network has a wide range of potential, practical and useful applications. Majority of such applications require an optimization to the communication among the sensors, so as to serve data in short latency and with minimal energy consumption. The sensor nodes in WSN are battery powered devices that consumes energy during data transmission and processing. Sink node send query messages in network, nodes may or may not reply back to the sinks query messages, but battery dissipates due to processing. Data transmission in WSN consumes more energy than processing therefore it is appropriate to exploit the benefits of caching. Cooperative caching is used for the reduction in the state of non utility of data and decreases the requirement of wireless bandwidth and energy. Cooperative caching which secure sharing of data among various nodes reduces the number of communications over the wireless channels and thus enhances the overall lifetime of a wireless sensor network. To overcome problem of cooperative cache a new technique is proposed which gives better results than existing one. Network simulator NS-2 is used for the simulations.
\end{abstract}

\section{Keywords}

WSN, Cooperative Caching, Cluster Head, CCL, CCF

\section{INTRODUCTION}

Wireless sensor network (WSN) [1] is a self healing and self organizing networks. Self healing network enable nodes to reconfigure their link associations and find other pathways around powered-down nodes or failed nodes. Self organizing allows a network automatically join new node without the need for manual interference. In other words, wireless sensor is a computer network which is composed of a large number of sensor nodes. Sensor nodes are those which are capable of sensing environment around them. Sensor nodes are devices which are capable of gathering, storing, sensing and transmitting information. Sensor nodes can be deployed anywhere without install it. The gathered information can be retrieved.

A wireless sensor network is collections of sensing device that can be wirelessly communicate. Each device is capable of talk to its peer, sense, process. It is centralized system. It is inexpensive to install and no wiring is required for data transfer. A wireless sensor network is a technology that provides monitoring. Wireless sensor play an important role in the processing of structural response data. In a wireless sensor networks nodes are organized in a cooperative manner [8].

\author{
Mritunjay Kumar Rai \\ Department of Electronics \& \\ Communication Engineering \\ Lovely Professional University Phagwara, Punjab
}

(India)

\begin{abstract}
Wireless sensor offers impressive computational resources for processing data. Wireless Sensor networks are self organizer and are deployed in ad-hoc systems. Each node in wireless sensor is consist of multiple types of memory and processing elements, RF transceiver, a power source, sensors and actuators. Wireless Sensor Network is a distributed real time systems.
\end{abstract}

Wireless Sensor Network is a latest technology that has an ability to coordinate a large network into a single network. It is also consist of large number of low cost devices. Wireless Sensor Network is a Light weighted distributed system. There is no need of external infrastructures in wireless sensor network to communicate with other devices. The sensor in wireless sensor networks deployed are self organized and multi hop in nature. The wireless sensor networks consume a lots of energy while transfer data from one device to other. There is need to adjust transfer and sensing rate to avoid energy consumption in wireless sensor networks [5].

Wireless Access Points: The wireless access point acts as the central unit between the wired and wireless network. The wireless access points are basically the base stations which are attached to the wired network.

Wireless Clients: Wireless clients are act as the network interface. The wireless clients are work with the various computer devices such as laptops, pc's that communicate with the access points. In clustering, the sensor nodes are partitioned into different clusters. Every cluster is superintended by a node referred as cluster head $(\mathrm{CH})$ and alternate nodes referred as cluster nodes. Cluster nodes don't communicate directly with the sink node. They need to pass the collected data to the cluster head [9], Cluster head will aggregate the data collected, received from cluster nodes and forward it towards the base station. Therefore reduces the energy consumption and various messages communicated to base station.

Further the paper is arranged as follows. Section 2 discussed on the cooperative caching schemes. Section 3 is discussed on the proposed methodology. In section 4 we have discussed the outputs and results of the proposed technique in comparison to the existed technique.

\section{COOPERATIVE CACHING}

Cooperative caching is a technique, in which various sensor nodes share and coordinate cache data to overcome communication cost and use the total cache space of cooperating sensors. The simple assumption we make, is that each sensor node has a moderate local storage capacity 
associated with it, i.e., a flash memory. Although, there exits flash memory with various gigabyte storage capability.

Every sensor node caches the frequently accessed data items in its non-volatile memory such as flash memory. The data items which are cached in the flash memory satisfy not only the node's own requests but also the data requests passing through it from other nodes. For a data miss in the local cache, the node first searches the data in its zone before forwarding the request to the next node that lies on a path towards the data source.

The cache admission control process is based on the distance criteria of a node from the sink and gives higher priority to the nodes located nearer to the sink [10], usefulness based data replacement policy has been maintained to ensure that more useful data is retained in the local cache of a node. Protocols are deviated from such approach.

\subsection{Cache Data}

In cache data middle nodes cache the data to operate future requests as a replacement for of fetching data from their source.

\subsection{Cache Path}

In cache path nodes that are mobile cache the data path and exploit it to redirect future requests to the close to node which has the data instead of the faraway origin node. Providing continuous information to mobile sinks with uninterrupted communication is a big challenge in designing large-scale sensor networks. Lots of research in data routing, data compression and in-network aggregation [2] has been proposed in recent years. If caching implemented optimally can reduce a lot of network traffic and help in providing higher data availability to the sink [6]. Each concentric circular cache layer gets a token which decides which cache layer will caches the data. The Circular Cache layers (CCL) are formed by the Circular Cache Formation (CCF) algorithm. The algorithm searches for the geographical coordinates of the nodes around the sink, by flooding request messages to determine their distance from the sink. A cache layer is forms when the nodes which fall under a particular distance from the sink [4], the discovery of cached data is operated by a simple cache discovery scheme. Finally, a data replacement policy is given which helps in removing obsolete data from the caches [3]. Cooperative caching plays an important role to handle the request. This helps to reduce non availability of data, reduce requirements for bandwidth and decided which data should be cached or not in wireless sensors networks.

\section{PROPOSED METHODOLOGY}

The wireless sensor networks is the application based network in which sensor nodes are used to sense the environmental conditions. The sink sends the query message to the sensor node and sensor node reply back to the query message. This approach is the energy inefficient technique. To reduce the battery consumption of the sensor networks cooperative caching scheme is used which increase networks lifetime.
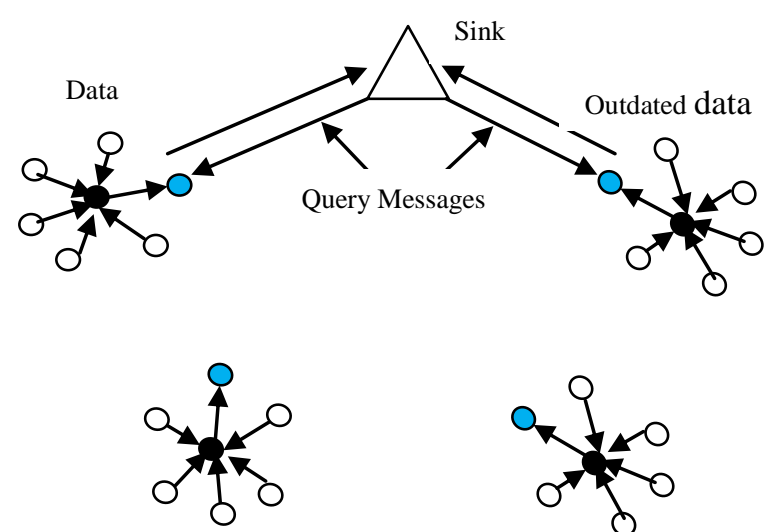

Cache Node

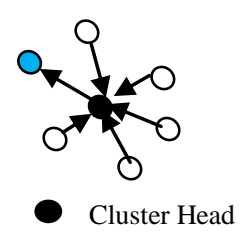

Fig 1: Proposed Scheme Scenario

In cooperative caching based network, caching nodes are selected on the basis of some assumptions. Sensor nodes are store data on cache. In cooperative caching based network, the sink will not flood the query message in whole network. It will send message only to the caching based network. The whole network will be divided into parts and in each part caching nodes are deployed as shown in Fig 1. The sink will send query message to its specific nearby caching nodes and that node will reply back with the query reply message. In cooperative caching based network, the problem of inconsistence can occur which reduces the network reliability. In this work, enhancement in the cooperative caching based technique is proposed which maintains consistence in the network. The proposed scheme is based on the pull and push based technique. In push based scheme, if the caching node has required data it will revert back to sink otherwise caching node will forward the query message to other its nearest caching node. The caching nodes which have required data will revert back to the sink. The caching node which sends revert back to sink, that caching node will sends the caching updated message to all other caching nodes in the network.

\section{RESULTS}

The whole scheme is implemented on the network simulator ns-2 [12] (version 2.35) in which NAM editor and X-graphs are used to show the simulations and to show the outputs in graphical form respectively for consistent network with respect to the inconsistent network. Network parameters that are used for our simulation purpose are as described below.

Table 1: Network Parameters

\begin{tabular}{|l|l|}
\hline Number of Nodes & 25 \\
\hline Antenna type & Omni-directional \\
\hline Queue & Priqueue \\
\hline Number of channels & 10 \\
\hline Propagation Modal & $\begin{array}{l}\text { Two Ray propagation } \\
\text { modal }\end{array}$ \\
\hline Physical medium & Wireless medium \\
\hline
\end{tabular}

AODV [11] routing protocol is used for our simulation purpose and with the help of X-graphs we have shown the output of the proposed scheme graphically for different parameters of networks energy consumption in the network, throughput of the network and the delay occurred in the network. 
In Fig 2 battery consumption of the sensor nodes is shown in the caching based network with inconsistence and battery consumption with proposed technique. The red line shows the battery consumption of the old scenario and green line shows the battery consumption of sensor nodes in proposed technique. The battery consumption of sensor nodes is reduced about $1 \%$ by the new scheme as a result of consistence is maintained between all the caching nodes and sink will receive latest data from all the caching nodes.

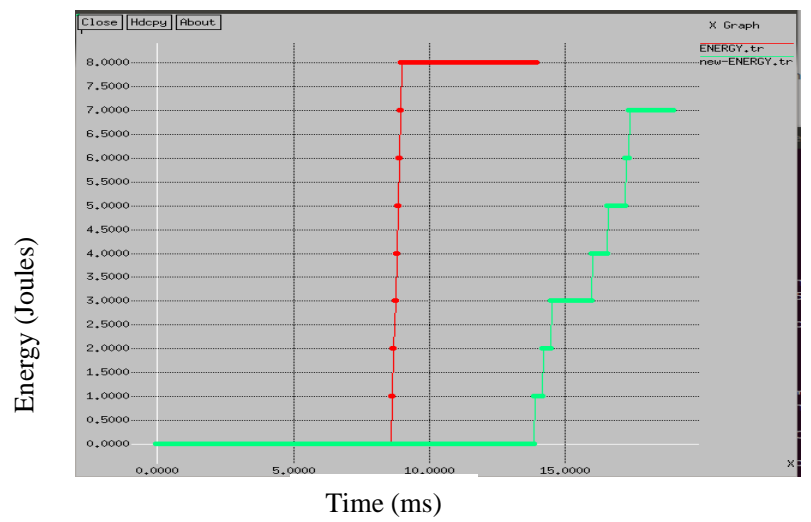

Fig 2: Output Graph for Energy

As illustrated in the Fig 3, the network throughput is shown which is increased in the new proposed technique as compared to the existing technique.

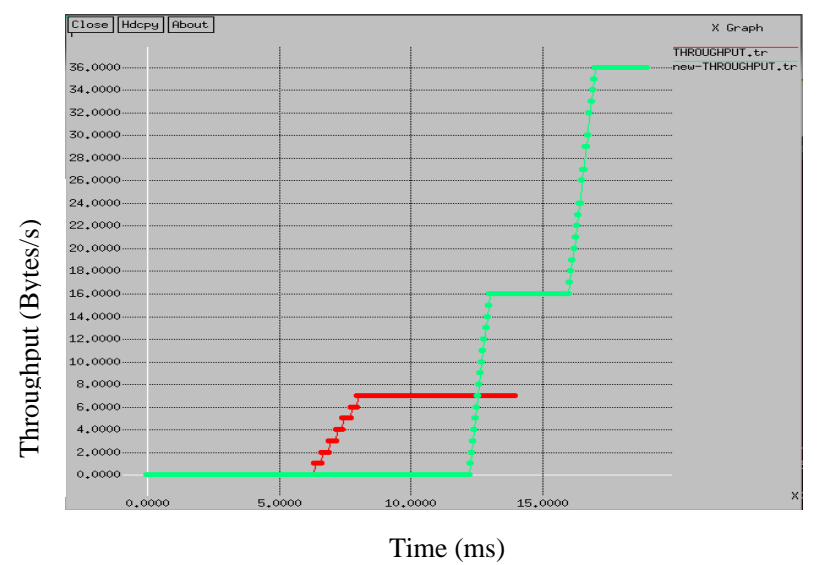

Fig 3: Output Graph for Throughput

Throughput of the new scenario has been increased by 4 times as consistence is maintained in the network.

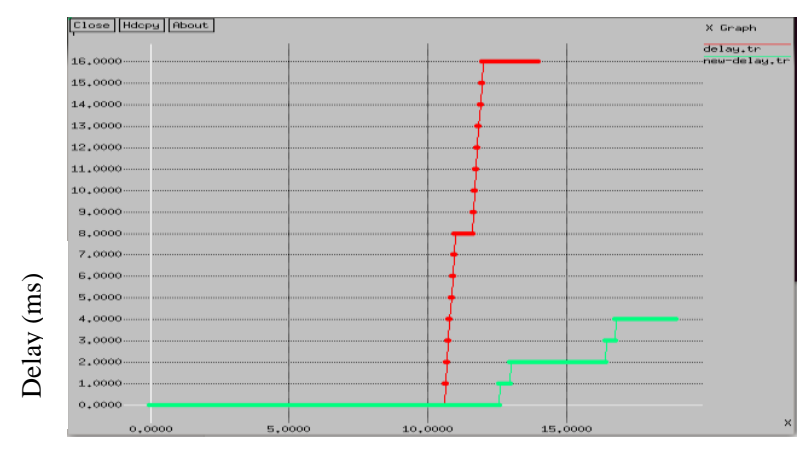

Time (ms)

Fig 4: Output Graph for Delay
Fig 4 illustrates delay comparison between the two scenarios proposed and the existed in which the new scenario have very less delay as comparison to the old preexisted scheme. From graphs it is quite clear that our proposed technique have 8 times less delay as compared to the existing technique, which increases the overall performance of our system and also increases the reliability of the system to great extent.

\section{CONCLUSION}

In this research paper, we conclude that battery consumption of sensor nodes is the major issue in the wireless network which reduces network lifetime. To reduce battery consumption of sensor nodes cooperative caching technique is very efficient technique. Due to inconsistence between caching nodes, network reliability is reduced. To maintain consistence between the caching nodes novel technique is been proposed in this paper which is based on push and pull based techniques. The experimental results shows that proposed technique increase the throughput by a factor of 4 times and delay is reduced by the factor of 8 times and energy consumption by factor of $1 \%$. The overall efficiency of the system is increased by the factor of $4.1 \%$, which increases the reliability of the network in the proposed technique defined network conditions. Proposed technique discussed in this paper will give benefit for various research scholars.

\section{ACKNOLEDGEMENT}

Especial thanks to my mentor cooperation along my research work accomplishment along with all my academic comembers and I would also thankful to the IJCA journal for providing us such a special opportunity for the publication of our research work.

\section{REFERENCES}

[1] I. F. Akyildiz, W. Su, Y. Sankarasubramaniam, and E.Cayirci, "A survey on sensor networks," IEEE Communications Magazine, Vol. 40 Issue. 8, pp. 102 114, August 2002.

[2] E. Ilker Oyman and Cem Ersoy,'Multiple Sink Network Design Problem in Large Scale Wireless Sensor".

[3] Md Ashiqur Rahman and Sajid Hussain, "Effective Caching in Wireless Sensor Network", 21st International Conference on Advanced Information Networking and Applications Workshops, IEEE Computer Society Washington, DC, USA, Vol.1, pp.43-47, 2007.

[4] Ms Manisha Rana \& Gurpreet Kaur, "Improved Circular Caching Based On WSN With Multi sink", International Journal Of Engineering Science \& Humanities, Vol. 2 Issue 2, pp. 1-4, June 2012.

[5] Nikos Dimokas, Dimitrios Katsaros, Yannis Manolopoulos, "Cooperative Caching in Wireless Multimedia Sensor Networks", Springer Science Business Media, LLC 2008".

[6] Sudhanshu Pant, Naveen Chauhan, Prashant Kumar, "Effective Cache based Policies in Wireless Sensor Networks: A Survey", International Journal of Computer Applications (0975 - 8887) Volume 11No.10, December 2010.

[7] Naveen Chauhan, L.K. Awasthi, Narottam Chand, "Global Cooperative Caching for Wireless Sensor 
Networks", IEEE World Congress on Information and Communication Technologies, pp.235-239, 2011.

[8] Narottam Chand, "Cooperative Data Caching in WSN", World Academy of Science, Engineering and Technology 63, pp.90-94, 2012.

[9] Amir Akhavan Kharazian, Kamal Jamshidi and Mohammad Reza Khayyambashi "Adaptive Clustering in Wireless Sensor Networks: Considering Nodes with Lowest Energy", International Journal of Ad hoc, Sensor \& Ubiquitous Computing Vol.3, No.2, April 2012.

[10] Wint Yi Poe, Jens B. Schmitt, "Sink Placement without Location Information In Large Scale Wireless
Sensor Networks", 5th ACM SIGCOMM Asian Internet Engineering Conference (AINTEC), Bnagkok, Thailand, November 2009.

[11] C.E. Perkins \& E. Royer, "Ad hoc on-demand distance vector routing", Proceedings of IEEE workshop on mobile computing systems and applications, 1999.

[12] Teerawat Issariyakul and Ekram Hossain, "Introduction to Network Simulator NS2", Springer Publication, 2009.

[13] Chia-Lin Yang and Alvin R., "Lebeck Push vs. Pull: Data Movement for Linked Data Structures", ACM International Conference on Supercomputing (ICS), 2000 . 